\section{OPEN ACCESS}

Edited by:

Kum Fai Yuen,

Nanyang Technological University, Singapore

Reviewed by:

Guanqiu Qi,

Chung-Ang University, South Korea

Xueqin Wang

Chung-Ang University, South Korea

${ }^{*}$ Correspondence:

S. M. Yasir Arafat arafatdmc62@gmail.com

Specialty section

This article was submitted to Public Mental Health,

a section of the journal

Frontiers in Public Health

Received: 08 September 2020

Accepted: 15 October 2020

Published: 05 November 2020

Citation:

Arafat SMY, Kar SK, Menon V Alradie-Mohamed A, Mukherjee S, Kaliamoorthy C and Kabir R (2020)

Responsible Factors of Panic

Buying: An Observation From Online Media Reports.

Front. Public Health 8:603894.

doi: $10.3389 /$ fpubh.2020.603894

\title{
Responsible Factors of Panic Buying: An Observation From Online Media Reports
}

\author{
S. M. Yasir Arafat ${ }^{1 *}$, Sujita Kumar Kar ${ }^{2}$, Vikas Menon ${ }^{3}$, Angi Alradie-Mohamed ${ }^{4}$, \\ Srijeeta Mukherjee ${ }^{5}$, Charanya Kaliamoorthy ${ }^{3}$ and Russell Kabir ${ }^{4}$ \\ ${ }^{1}$ Department of Psychiatry, Enam Medical College and Hospital, Dhaka, Bangladesh, ${ }^{2}$ Department of Psychiatry, King \\ George's Medical University, Lucknow, India, ${ }^{3}$ Department of Psychiatry, Jawaharlal Institute of Postgraduate Medical \\ Education and Research, Puducherry, India, ${ }^{4}$ School of Allied Health, Faculty of Health, Education, Medicine, and Social \\ Care, Anglia Ruskin University, Chelmsford, United Kingdom, ${ }^{5}$ Department of Psychiatry, Maharaja Krushna Chandra \\ Gajapati Medical College, Brahmapur, India
}

Background: Panic buying is an erratic human behavior that has been reported irregularly and episodically. There is a dearth of studies exploring the identifiable factors accounting for it. We aimed to identify the factors responsible for panic buying extracted from online media reports.

Methods: We scrutinized the media reports published in English discussing the different aspects of panic buying. We collected data until May 30, 2020, and searched the possible mentioned reasons responsible for panic buying.

Results: We analyzed a total of 784 media reports. The majority of the reports were found in Bing (18\%), Ecosia (12.6\%), Google (26.4\%), and Yahoo (12.5\%). Panic buying was reported in 93 countries. Among the 784 responses, a total of 171 reports did not explain the responsible factors of panic buying. Therefore, we analyzed the remaining 613 reports to identify the same. A sense of scarcity was reportedly found as the important factor in about $75 \%$ of the reports followed by increased demand (66.07\%), the importance of the product (45.02\%), anticipation of price hike (23.33\%), and due to COVID-19 and its related factors (13.21\%). Other reported factors were a rumor, psychological factors (safety-seeking behavior, uncertainty, anxiety reduction, and taking control), social learning, lack of trust, government action, and past experience.

Conclusions: The study revealed the responsible factors of panic buying extracted from media reports. Further, studies involving the individuals indulging in panic buying behavior are warranted to replicate the findings.

Keywords: panic buying, media report analysis, content analysis, COVID-19, pandemic

\section{INTRODUCTION}

Panic buying $(\mathrm{PB})$ is an erratic human behavior that has been reported irregularly and episodically; however, $\mathrm{PB}$ has been reported since long before and appeared especially during a major emergency event (1-3). It has been noticed and reported in online media during this COVID-19 pandemic in several countries in the world $(1,4,5)$. It has been explained as "the phenomenon of a sudden 
increase in buying of one or more essential goods in excess of regular need provoked by adversity, usually a disaster or an outbreak resulting in an imbalance between supply and demand" (4). A group of authors speculatively described it as a manifestation of underlying conflict and uncertainty during the pandemic, a way of coping with a stressful condition, gaining control, and social pressure to conform to alike behaviors (5). Theoretically, few mental processes have been discussed such as the perception of scarcity of necessary goods, way to gaining control, uncertainty, insecurity, herd behavior, primitive behavior, media influence, and lack of confidence in authorities (6). A recent systematic review also revealed some psychological factors responsible for $\mathrm{PB}$ mentioned such as (a) perceived threat and scarcity of the goods, (b) fear of the unknown resulting from negative emotions and uncertainty, (c) coping behavior such as anxiety reduction and gaining control, and (d) socialpsychological issues (7). Singh and Rakshit mentioned PB as herd behavior (8) and Tsao et al. mentioned supply chain disruption (9). Again, Chen et al. (2) described that disturbed judgments resulting from improper information during a crisis are responsible for $\mathrm{PB}$ where authors tried to explain it with economics and psychology theories. The endowment effect and commodity and prospect theories have been proposed to explain PB based on economics (2). Additionally, the authors hypothesized three mental processes mentioning autonomy, relatedness, and competence as attributing factors for PB (2).

Although newer studies are coming out, there is a dearth of empirical studies exploring the identifiable factors responsible for it. However, there are also challenges to studying the phenomenon systematically as it is erratic, irregular, episodic, sudden, unpredictable, and mostly happens during emergency situations (10). Interestingly, PB is a newsworthy issue and has been frequently reported $(1,4)$. Therefore, we aimed to evaluate the responsible factors of $\mathrm{PB}$ extracted from online media reports.

\section{MATERIALS AND METHODS}

\section{Study Setting and Data Collection}

This was a prospective analysis of 18 internet search engines (Table 1), all of which were identified a priori by the team of investigators. Three different investigators equally divided the search engines and simultaneously carried out the search using only the term "panic buying"; no combination was used to minimize missing media report that discussed PB. We scrutinized the media reports published in English discussing the different aspects of PB with special attention to attributable factors of it. We searched and collected data from 1 to 31 May 2020 in Google Form. Data were collected from any report from the media covering the blogs, personal views, opinions, and news. We excluded social media posts as social media posts are more of personal views and very often emotionally biased. People may post/repost things that are trending just to appear active online without really understanding its impact. Conformity bias and groupthink could act as other sources of potential biases. Subsequently, two other investigators did
TABLE 1 | Search details.

\begin{tabular}{lcc}
\hline Search engine & $\boldsymbol{n}$ & $\%$ \\
\hline Aol.com & 28 & 3.6 \\
Baidu & 6 & 0.8 \\
Bing & 141 & 18.0 \\
Duckduckgo & 36 & 4.6 \\
Ecosia & 99 & 12.6 \\
Exalead & 11 & 1.4 \\
Excite & 13 & 1.7 \\
Gigablast & 4 & 0.5 \\
Google & 207 & 26.4 \\
Lycos & 3 & 0.4 \\
Mojeek & 8 & 1.0 \\
Qwant & 9 & 1.1 \\
Startpage.com & 52 & 6.6 \\
Swisscows & 28 & 3.6 \\
Webcrawler & 13 & 1.7 \\
Yahoo & 98 & 12.5 \\
Yandex & 14 & 1.8 \\
Yippy & 14 & 1.8 \\
\hline Total & 784 & 100 \\
\hline
\end{tabular}

cross-checks of the data and data cleaning. Duplications were checked and removed by tracing the date of publication and title of the reports. Data collectors were well trained through frequent Zoom meetings by the team of investigators before starting the study. Doubts on whether to include a report were sorted out by mutual discussion with a senior author (SMYA, SKK). In case they were unable to reach an agreement, the issue was resolved by involving all authors in a group discussion. The above methodology was adapted from previous media-based studies on PB that used a single keyword search strategy $(1,4)$.

\section{The Instrument}

Based on the existing literature $(3,5,6)$ and our previous works $(1,4)$, the team formulated the questionnaire through the Zoom meeting. The instrument had two sections consisting of (a) the identification section and (b) the attributed factors section. The identification section comprised the name of the country from where the report was published, the name of the country to which the report referred, type of newspaper, name of the newspaper, dates of publication, and the primary scarce object for PB. All the reports were scrutinized by careful reading, and all the mentioned attributable factors were identified and documented in the second section. We aimed to identify all the possible factors and the majority of the reports discussed several factors.

\section{Statistical Analysis}

We used simple descriptive statistics (frequency and percentages) to depict extraction such as the number of relevant reports identified from different search engines as well as various reasons for $\mathrm{PB}$. A word cloud analysis was used to summarize 


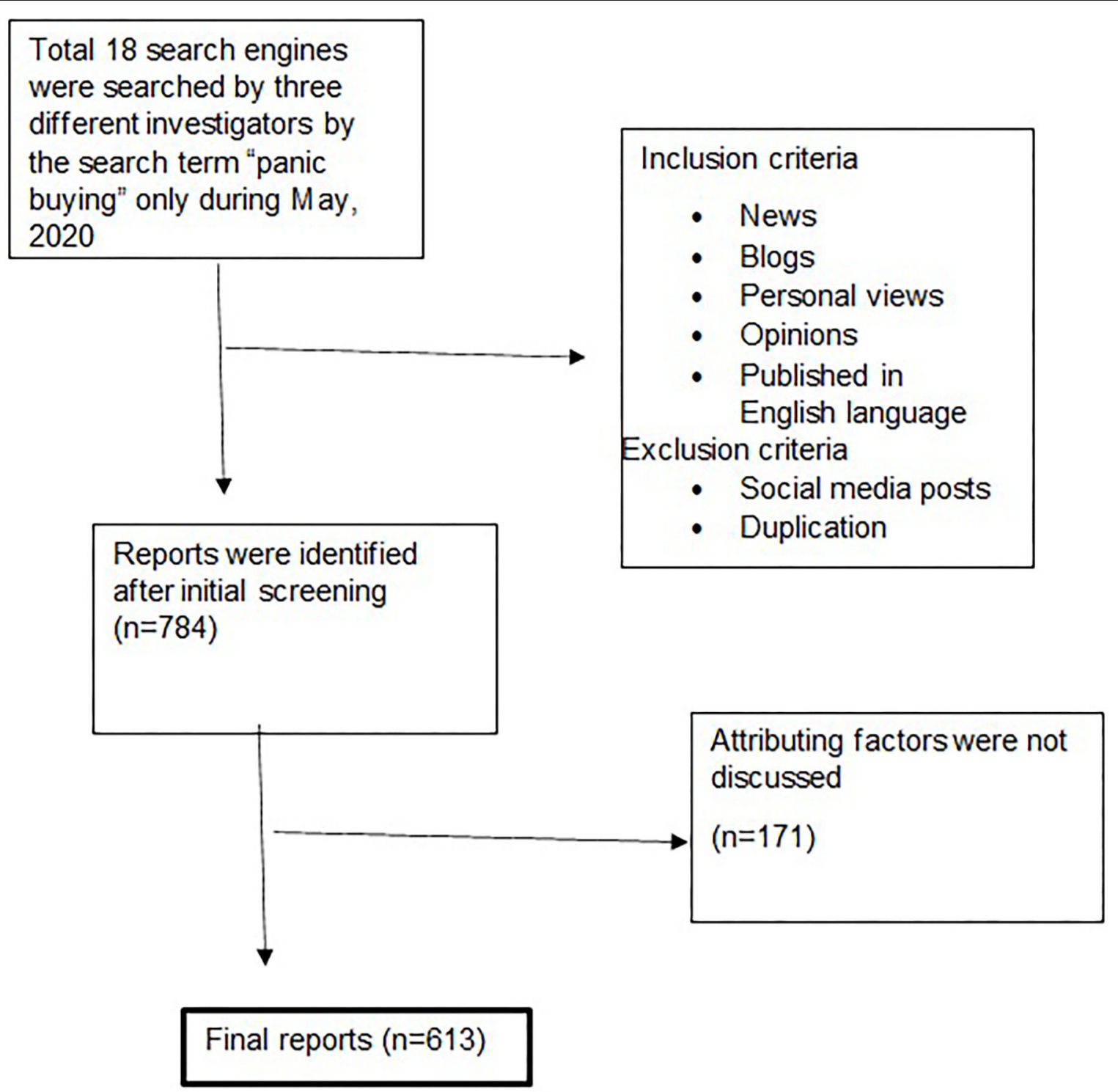

FIGURE 1 | Flow chart showing study sample selection.

search results in terms of frequency of $\mathrm{PB}$ reports from different countries.

\section{Ethics Statement}

The study was conducted complying with the declaration of Helsinki (1964). As we analyzed the publicly available media reports, no formal ethical approval was obtained.

\section{RESULTS}

We analyzed a total of 784 media reports (Figure 1). The majority of the reports were found in Bing (18\%), Ecosia (12.6\%), Google (26.4\%), and Yahoo (12.5\%) (Table 1). PB was reported in 93 countries (Figure 2). Among the 784 responses, a total of 171 reports did not explain the responsible factors of $\mathrm{PB}$. Therefore, we analyzed the rest 613 reports to identify the responsible factors of $\mathrm{PB}$. We considered extracting as many as possible attributing factors from the contents. Therefore, a single report had multiple responses when the factors were segregated. A sense of scarcity was reportedly found as the important responsible factor of PB that was found in about $75 \%$ of the reports $(n=$ $456)$ followed by increased demand $(66.07 \%)$, the importance of the product $(45.02 \%)$, anticipation of price hike $(23.33 \%)$, and due to COVID-19 and its related factors (13.21\%). The rumor was mentioned as responsible factors in 53 reports $(8.65 \%)$. We considered safety-seeking behavior $(n=5)$, uncertainty $(n=$ $6)$, anxiety reduction $(n=24)$, and taking control $(n=15)$ as psychological factors, which constituted $8.16 \%(n=50)$ of reports. Other reported factors were social learning, lack of trust, government action, and past experience (Table 2). 


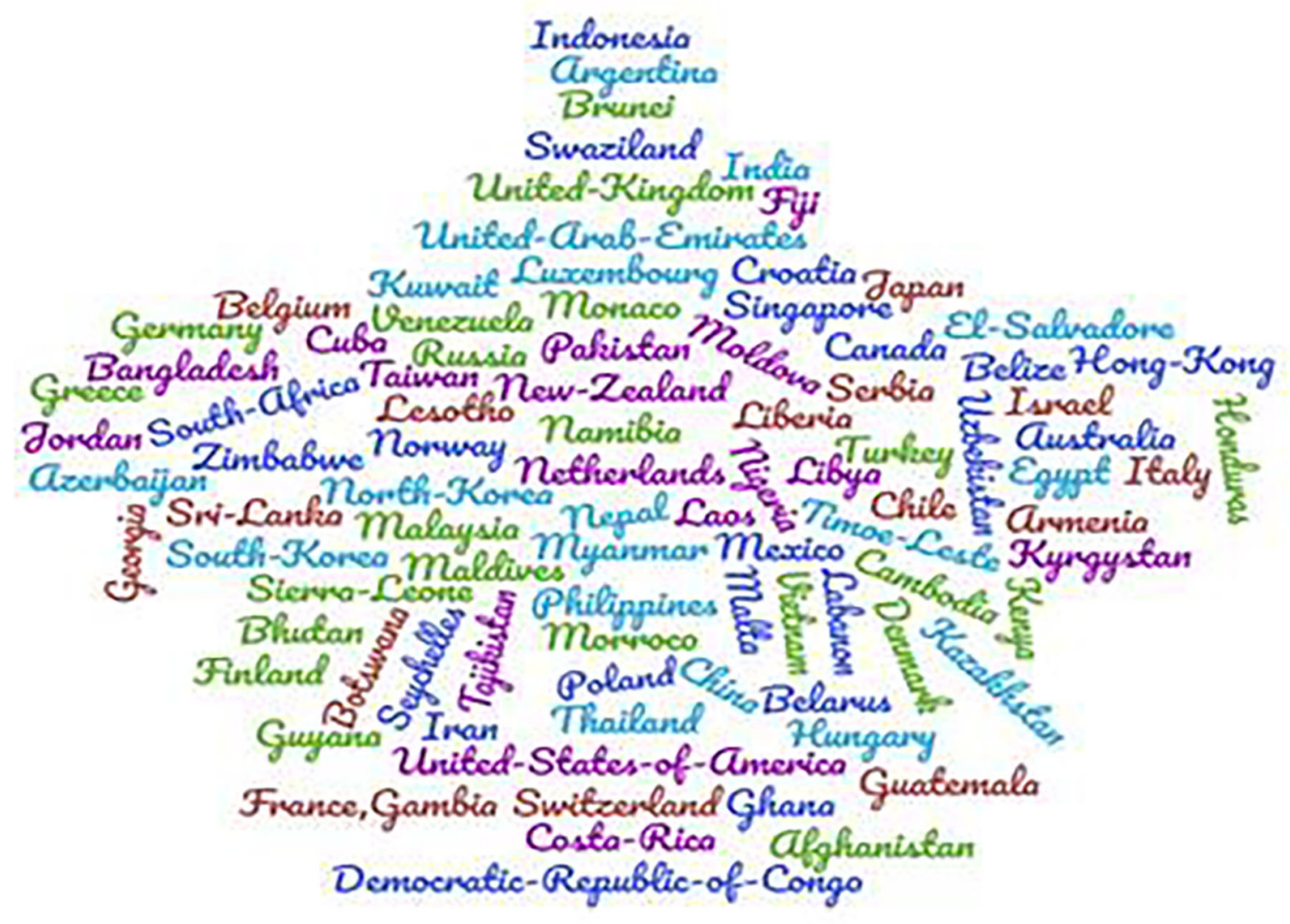

FIGURE 2 | Word cloud showing countries reporting panic buying behavior.

TABLE 2 | Reasons of panic buying $(n=613)$.

\begin{tabular}{lcc}
\hline Reasons of PB & $\boldsymbol{n}$ & $\%$ \\
\hline Scarcity & 456 & 74.39 \\
Increased demand & 405 & 66.07 \\
Necessary goods & 276 & 45.02 \\
Anticipated price hike & 143 & 23.33 \\
COVID-19, lockdown, planned, & 81 & 13.21 \\
Rumor & 53 & 8.65 \\
Psychological & 50 & 8.16 \\
Social learning & 15 & 2.45 \\
Lack of trust & 6 & 0.98 \\
Government's action & 6 & 0.98 \\
Past experience & 3 & 0.49 \\
\hline Total & 613 & 100 \\
\hline
\end{tabular}

\section{DISCUSSION}

$\mathrm{PB}$ is a contemporary issue with a dearth of empirical studies regarding the responsible factors behind the behavior. We aimed to evaluate the responsible factors of $\mathrm{PB}$ extracted from online media reports. The study revealed that $\mathrm{PB}$ has been reported in
93 countries. Previous studies reported the distribution of the countries; however, none of the studies mentioned such wide distributions $(1,2,4,5)$.

The study revealed several responsible factors, i.e., a sense of scarcity, increased demand, the importance of the goods, the anticipation of price hike, COVID-19 pandemic, rumor, safety-seeking behavior, uncertainty, anxiety reduction, taking control, social learning, lack of trust, government action, and past experience as the responsible factors for PB (Table 2). A high rate attribution was mentioned in reports among few factors such as perceived scarcity $(75 \%)$, increased demand (66\%), the importance of the product (45\%), and anticipation of price hike (23\%). The COVID-19 pandemic and related issues were attributed to about $13 \%$ of the reports. The rumor was mentioned as responsible factors in 53 reports $(8.65 \%)$. A recent systematic review identified the factors grossly in four domains, namely, perception, fear of the unknown, coping strategy, and psychosocial factor (7). The authors also subdivided the gross areas into different parts. Others mentioned the responsible factors as a mismatch between routine work and uncertainty, coping strategy, gaining control, social learning, and supply chain disruption $(5,8,9)$. It can also be due to any perceived or real external threat and/or own nervousness (11). 
Perceived scarcity has been mentioned repeatedly by different groups of authors as an important responsible factor for PB $(2,6,7)$. However, perception of threat and/or perception of risk have also been mentioned as a factor that has not been found in the current study $(3,7)$. We speculate that there might have been some overlaps between the perception of risk and our included psychological factor, which covers safety-seeking behavior, uncertainty, anxiety reduction, and taking control.

The second most important attributing factor has been identified as increased demand that was mentioned in about $66 \%$ of the responses. It is quite interesting, and it could be a result rather a causative factor because in a normal equilibrium, the demand should not be increased until an adverse and/or precipitating event occurs. It can be explained by taking consideration of the proposed definition by Arafat et al. (4). The phenomenon starts with a sudden increase in buying precipitated by adversity, usually a disaster or an outbreak resulting in a shortage of supply (4). The supply shortage can precipitate the rise of demand (9). Furthermore, the shortage of necessary goods is widely circulated by the media, which in turn creates insecurity, uncertainty, and more PB. Therefore, increasing demand can be explained by the precipitating events and results of the dissemination of shortage news.

The third important identified factor is the importance of the product that has been attributed to about $45 \%$ of the reports. It is quite plausible because PB mostly happens in cases of essential goods that are supposed to be used in the immediate future. Previous studies also mentioned similar factors $(4,5)$.

The fourth important identified factor is the anticipation of the price hike that was attributed to about $23 \%$ of the reports. A complex interaction should be warranted in case of anticipation of price hike, because the precipitating event such as the COVID-19 pandemic, perception regarding the supply chain, media propagation, rumors, and personal experience may have interaction to bear the perception of price hike (2).

Other responsible factors are the COVID-19 pandemic, rumor, safety-seeking behavior, uncertainty, anxiety reduction, taking control, social learning, lack of trust, government action, and past experience as the responsible factors for PB (Table 2). All the factors could explain the previous explanations $(2-7,12-14)$.

\begin{tabular}{|lll|}
\hline Primary factor & Secondary factors & Tertiary factors \\
\hline
\end{tabular}

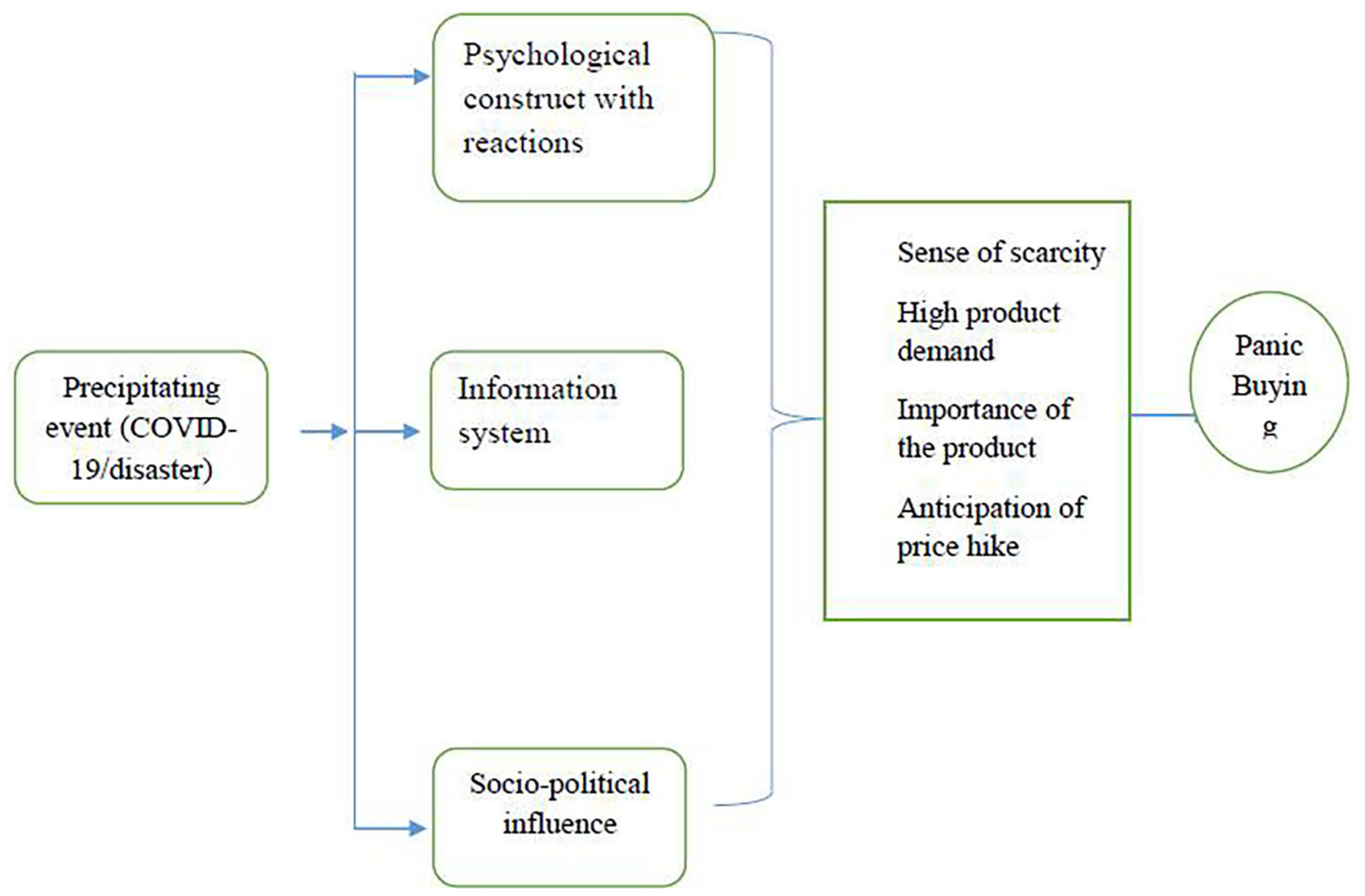

FIGURE 3 | Causal model of panic buying. 
Here, we theorized the adverse or precipitating event as the primary causative factor; psychological construct with reactions, social structure, and information system are the secondary factors; and subsequently, other factors such as sense of scarcity, increased demand, the importance of the product, and anticipation of the price hike are the tertiary factors (Figure 3). There is a complex constant interaction between the primary, secondary, and tertiary factors.

\section{Strengths of the Study}

There is a dearth of empirical studies exploring different aspects of $\mathrm{PB}$. To the authors' best knowledge, this is the first empirical study exploring the responsible factors of the behavior.

\section{Limitations}

The current study has several limitations. Everyone should be aware of the scientific quality of data as we analyzed any sort of media reports that may not be considered as scientific data. We also included only media reports published in English and excluded reports published in other languages. We only searched with a single keyword, "panic buying," without considering the synonyms, which may reduce the number of reports.

\section{CONCLUSION}

The study revealed the factors responsible for $\mathrm{PB}$ extracted from media reports, which include a sense of scarcity, increased demand, the importance of the product, the anticipation of price hike, COVID-19, rumor, psychological factors (safety-seeking behavior, uncertainty, anxiety reduction, taking control), social learning, lack of trust, government action, and past experience.

\section{REFERENCES}

1. Arafat SMY, Kar SK, Menon V, Marthoenis M, Sharma P, Alradie-Mohamed A, et al. Media portrayal of panic buying: a content analysis of online news portals. Glob Psychiatry. (2020) 3:249-54. doi: 10.2478/gp-2020-0022

2. Chen Y, Rajabifard A, Sabri S, Potts KE, Laylavi F, Xie Y, et al. A discussion of irrational stockpiling behaviour during crisis. J Saf Sci Resilience. (2020) 1:57-8. doi: 10.1016/j.jnlssr.2020.06.003

3. Bonneux L, Van Damme W. An iatrogenic pandemic of panic. BMJ. (2006) 332:786-8. doi: 10.1136/bmj.332.7544.786

4. Arafat SMY, Kar SK, Menon V, Kaliamoorthy C, Mukherjee S, AlradieMohamed A, et al. Panic buying: an insight from the content analysis of media reports during COVID-19 pandemic. Neurol Psychiatry Brain Res. (2020) 37:100-3. doi: 10.1016/j.npbr.2020.07.002

5. Sim K, Chua HC, Vieta E, Fernandez G. The anatomy of panic buying related to the current COVID-19 pandemic. Psychiatry Res. (2020) 288:113015. doi: 10.1016/j.psychres.2020.113015

6. Arafat SMY, Kar SK, Marthoenis M, Sharma P, HoqueApu E, Kabir R. Psychological underpinning of panic buying during pandemic (COVID-19). Psychiatry Res. (2020) 289:113061. doi: 10.1016/j.psychres.2020.113061

7. Yuen KF, Wang X, Ma F, Li KX. The psychological causes of panic buying following a health crisis. Int J Environ Res. (2020) 17:3513. doi: 10.3390/ijerph17103513

8. Singh CK, Rakshit PA. Critical Analysis to comprehend Panic buying behaviour of Mumbaikar's in COVID-19 era. Studies in Indian Place Names. (2020) 40:44-51.

9. Tsao YC, Raj PVRP, Yu, V. Product substitution in different weights and brands considering customer segmentation and panic buying behavior.
The extracted factors can be theoretically explained based on the previous reports revealing a complex interaction among a precipitating stimulus, personality construct, and environmental influences. Practically, adequate actions targeting the reasons could be beneficial for the prevention of PB. Preparedness for future episodes should have a special focus on the identified factors to reduce the panic among the general population. Further, empirical studies involving the individuals indulging the PB behavior are warranted to replicate the findings and/or nullify it. Qualitative studies could be a potential option to explore the psychological aspects responsible for the behavior.

\section{DATA AVAILABILITY STATEMENT}

The raw data supporting the conclusions of this article will be made available by the authors, without undue reservation.

\section{ETHICS STATEMENT}

The study was conducted complying with the declaration of Helsinki (1964). As we analyzed the publicly available media reports, no formal ethical approval was obtained.

\section{AUTHOR CONTRIBUTIONS}

SA contributed to the idea, concept, design, data analysis, and writing. SK and RK contributed to the concept. VM contributed to concept, design, and writing. AA-M, SM, and CK contributed to the data enumeration. All authors contributed to the article and approved the submitted version.
Ind Mark Manag. (2019) 77:209-20. doi: 10.1016/j.indmarman.2018. 09.004

10. Arafat SMY, Kar SK, Shoib S. Panic buying: is it really a problem? Int J Soc Psych. (in press) (2020) doi: 10.1177/0020764020962539

11. Harmon D, Lagi M, de Aguiar MA, Chinellato DD, Braha D, Epstein IR, et al. Anticipating economic market crises using measures of collective panic. PLoS ONE. (2015) 10:e0131871. doi: 10.1371/journal.pone.0131871

12. Arafat SMY, Kar SK, Kabir R. Possible controlling measures of panic buying during COVID-19. Int $J$ Ment Heal Addict. (2020). doi: 10.1007/s11469-020-00320-1. [Epub ahead of print].

13. Ahmad AR, Murad HR. The impact of social media on panic during the COVID-19 pandemic in Iraqi Kurdistan: online questionnaire study. J Med Internet Res. (2020) 22:e19556. doi: 10.2196/19556

14. Hossain MS, Ferdous S, Siddiqee MH. Mass panic during Covid-19 outbreakA perspective from Bangladesh as a high-risk country. J Biomed Anal. (2020) 3:1-3. doi: $10.30577 /$ jba.v3i2.40

Conflict of Interest: The authors declare that the research was conducted in the absence of any commercial or financial relationships that could be construed as a potential conflict of interest.

Copyright () 2020 Arafat, Kar, Menon, Alradie-Mohamed, Mukherjee, Kaliamoorthy and Kabir. This is an open-access article distributed under the terms of the Creative Commons Attribution License (CC BY). The use, distribution or reproduction in other forums is permitted, provided the original author(s) and the copyright owner(s) are credited and that the original publication in this journal is cited, in accordance with accepted academic practice. No use, distribution or reproduction is permitted which does not comply with these terms. 